\title{
DANOS MECÂNICOS E QUALIDADE FISIOLÓGICA DE SEMENTE DE SOJA COLHIDA PELO SISTEMACONVENCIONALE AXIAL ${ }^{1}$
}

\author{
MARIACELESTEMARCONDES², EDISONMIGLIORANZA ${ }^{3}$, INÊS CRISTINABATISTADEFONSECA ${ }^{4}$
}

\begin{abstract}
RESUMO - Neste trabalho, objetivou-se avaliar dois tipos de colhedoras, a de sistema convencional ou de cilindro côncavo e a axial, quanto à qualidade fisiológica de sementes de duas cultivares de soja (Glycine max (L.)Merrill), colhidas em três períodos diferentes do dia. A colhedora de cilindro côncavo trabalhou, deslocando-se a 5,0km.h ${ }^{-1}$ e cilindro trilhador com 750 rotações por minuto (rpm). A colhedora de fluxo axial deslocou-se a $8,0 \mathrm{~km} \cdot \mathrm{h}^{-1} \mathrm{e}$ rotor com 650 rotações por minuto (rpm). As cultivares de soja utilizadas foram BRS 184 e BRS 133. Para a avaliação da qualidade de sementes foram conduzidos os testes de germinação, vigor, tetrazólio, danos mecânicos e a determinação do grau de umidade. Sementes provenientes de ambas as colhedoras apresentaram resultados estatisticamente similares em relação a danos mecânicos e qualidade fisiológica. Há variações no grau de umidade da semente de soja durante o dia, podendo, ao final da tarde (18 horas), apresentar-se inferior ao início da colheita (10 horas), em até 3 pontos percentuais. Tanto a colhedora de cilindro côncavo como a axial, desde que convenientemente utilizadas na colheita quanto às especificações de regulagem, não provocam diferenças na qualidade fisiológica da semente de soja.
\end{abstract}

Termos para indexação: Glycine max, colhedoras, qualidade de sementes.

\section{MECHANICAL DAMAGE AND FHYSIOLOGICAL SEED QUALITY OF SOYBEN WITH CONVENTIONALAND AXIAL FLOW HARVEST}

\begin{abstract}
This experiment aimed to evaluate two types of harvest combines, on seed quality of two soybean cultivars harvested at three times of - day. The conventional combine worked moving at 5,0km.h ${ }^{-1}$, cylinder speed 750 rotations per minute (rpm). The axial flow combine worked moving at $8,0 \mathrm{~km} \cdot \mathrm{h}^{-1}$, rotor speed 650 rotations per minute (rpm). The soybean cultivars used were BRS 184 and BRS 133. The germination test, vigor test, tetrazolium, mechanical damages and humidity test were used to evaluate the seed quality. Both harvest combines did not present statistical differences in mechanical damage and physiological quality. The seed moisture content varied during the day, but the results confirmed that it was always in a range of ideal moisture for the soybean mechanical harvest. Therefore the mechanical damage was controlled and consequently the seeds had good physiological quality regardless of the harvest combine used. There were variations in the soybean seed moisture content during the day, that could be lower by up to three percentage points at the end of the afternoon (6 p.m.) than at the start of harvesting (10 a.m.). Neither the conventional nor the axial flow combine harvesters, when used with specific adjustments, caused differences in soybean seed physiological quality.
\end{abstract}

Index terms: Glycine max, harvest combine, seed quality.

\footnotetext{
${ }^{1}$ Submetido em 29/03/2004. Aceito para publicação em 16/09/2004.

${ }^{2}$ Eng $^{\text {a }}$ Agr ${ }^{\mathrm{a}}$ mestranda em Agronomia, (UEL) - Universidade Estadual de Londrina, PR. celeste@pr.gov.br

${ }^{3}$ Eng $^{\circ}$ Agr $^{\circ}$ Dr., professor do Departamento de Agronomia, (UEL) -
}

Universidade Estadual de Londrina, PR. emiglior@uel.br

${ }^{4}$ Eng $^{\mathrm{a}}$ Agr $^{\mathrm{a}}$ Dr $^{\mathrm{a}}$, professora do Departamento de Agronomia, (UEL) Universidade Estadual de Londrina, PR. inescbf@uel.br 


\section{INTRODUÇÃO}

A crescente modernização da agricultura brasileira tem exigido dos diferentes segmentos mudanças profundas no sentido de racionalização do processo produtivo. Dentre os insumos do setor agrícola, a semente de alta qualidade ocupa papel fundamental em todo sistema de produção que vise à otimização de padrões quantitativos e qualitativos (Costa et al., 2001).

A qualidade das sementes é garantida através de padrões mínimos de germinação, purezas física e varietal, bem como sanidade, exigidos por normas de produção e comercialização estabelecidas e controladas pelo governo.

O processo de produção de sementes exige tecnologias que abrangem seleção da área, uso de variedades recomendadas, semeadura em épocas estabelecidas, acompanhamento do desenvolvimento vegetativo, tratos culturais, tratamentos fitossanitários, determinação do momento ideal de colheita e limpeza de maquinários, colhedoras e caminhões de transporte, que devem ser rigorosamente seguidas. A colheita constitui importante etapa no processo produtivo da soja, principalmente pelos riscos aos quais está sujeita a lavoura destinada à produção de sementes (Embrapa, 2002).

A colheita mecânica e o beneficiamento são as principais fontes de danos mecânicos em sementes. Na colheita, a semente fica particularmente susceptível ao dano mecânico, imediato ou latente (Paiva et al., 2000). Nesse caso, o dano mecânico ocorre no momento da debulha, isto é, no momento em que forças consideráveis são aplicadas sobre as sementes, a fim de separá-las da estrutura que as contêm. Ocorre, essencialmente, em conseqüência dos impactos recebidos do cilindro trilhador, no momento em que passam pelo côncavo. A semente na colhedora é um corpo estático, contra o qual se movimenta um corpo metálico, as barras do cilindro trilhador (Carvalho e Nakagawa, 2000).

O mercado dispõe, para a colheita mecânica de soja, de colhedoras com sistema de trilha com cilindro e côncavo transversais e, recentemente, as de fluxo axial, que podem produzir efeitos diferenciados na qualidade fisiológica de material a ser utilizado como semente (Marcos e Mielii, 2003).

Considerando a importância dos danos mecânicos e sua freqüente ocorrência no processo de produção de sementes, objetivou-se, com este trabalho, verificar o efeito de dois tipos de colhedoras na qualidade física e fisiológica de sementes de soja.

\section{MATERIAL E MÉTODOS}

Este estudo foi conduzido no município de Marilândia do Sul, Norte do Paraná, na safra 2002/03, com amostragem em campos de sementes, de produtor registrado junto ao órgão governamental responsável pelo processo de fiscalização, sementes da classe fiscalizada e certificada, das cultivares BRS 184 e BRS 133. As sementes foram amostradas em seis dias consecutivos e, em três horários diários, 10, 14 e 18 h.

Foram utilizados dois tipos de colhedoras, sendo uma do tipo cilindro côncavo e a outra, do tipo axial, com as seguintes regulagens: colhedora do tipo cilindro com côncavo - deslocamento a 5,0km.h ${ }^{-1}$, com velocidade periférica do molinete girando em velocidade $5 \%$ superior à da colhedora, cilindro trilhador com 750rpm e côncavo com abertura de $10 \mathrm{~mm}$ de entrada e $10 \mathrm{~mm}$ de saída; colhedora do tipo axial deslocamento a $8,0 \mathrm{~km} \cdot \mathrm{h}^{-1}$, com velocidade periférica do molinete girando em velocidade $10 \%$ superior à da colhedora, rotor com 650rpm, abertura do côncavo 3 a 4 na escala da colhedora e ventilador a 1050rpm na peneira, para limpeza da semente.

Foram realizadas as seguintes avaliações:

Grau de umidade no campo - o grau de umidade das sementes foi obtido com o equipamento de marca Dole 400, em amostras de sementes coletadas no tanque do graneleiro da colhedora, à medida que a máquina se deslocava em cada bloco especificado.

Coleta de amostras de sementes em nível de campo - para a avaliação das qualidades física e fisiológica foram coletadas amostras de $2 \mathrm{~kg}$ de sementes do tanque graneleiro, correspondente a cada dia especificado, para cada cultivar.

As análises de germinação, vigor, danos mecânicos e umidade das sementes amostradas foram realizadas em Laboratório Oficial da CLASPAR - Empresa de Classificação do Paraná, localizada em Maringá - PR.

Germinação - o teste foi realizado utilizando-se 400 sementes, semeadas em rolo de papel-toalha, marca Germitest, e colocadas em germinador regulado à temperatura constante de $25^{\circ} \mathrm{C}$. Considerou-se apenas, os resultados da primeira contagem do teste padrão de germinação, efetuada cinco dias após sua instalação, conforme as Regras para Análise de Sementes (Marcos Filho et al., 1987; Brasil, 1992).

Envelhecimento acelerado - foram utilizadas 400 sementes por tratamento, distribuídas em oito repetições de 50 sementes e acondicionadas sobre tela dentro de caixa 
plástica, tipo gerbox, contendo 40mL de água destilada. Depois de tampadas, as caixas foram colocadas em estufa incubadora, tipo BOD, à temperatura de $42^{\circ} \mathrm{C}$ por 48 horas (Marcos Filho et al., 1985). Após o envelhecimento, as sementes foram colocadas para germinar, seguindo a mesma metodologia adotada no teste de germinação.

Grau de umidade no laboratório - foi determinado pelo método expedito, com aparelho universal.

Teste de tetrazólio - conduzido com 400 sementes de cada cultivar, as quais ficaram pré-condicionadas em papel Germitest umedecido, com quantidade de água equivalente a 2,5 vezes o seu peso, durante 16 horas, em temperatura ambiente. Passado esse período, as sementes foram colocadas em Becker, numa solução de concentração de 0,075\% de 2,3,3-trifenil-cloreto-de-tetrazólio e, em seguida, foram colocadas no escuro, em estufa, com temperatura entre 35 e $40^{\circ} \mathrm{C}$, por três horas, para o desenvolvimento da coloração. Após a lavagem em água corrente, as sementes foram analisadas individualmente, verificando-se a percentagem de sementes viáveis, níveis de vigor e danos mecânicos, conforme metodologia descrita por França Neto et al. (1998).

Utilizou-se o delineamento em blocos casualizados, representados pelos dias de colheita, em esquema fatorial 2x3 (máquina x horário de colheita), com três e quatro repetições, respectivamente para as cultivares, BRS 184 e BRS 133.

Os dados foram submetidos à análise de variância e as médias comparadas pelo teste de Tukey ao nível de $5 \%$ de probabilidade (Banzatto e Kronka, 1992).

\section{RESULTADOS E DISCUSSÃO}

Sistematicamente, a bibliografia tem mostrado os efeitos negativos do baixo grau de umidade da semente (inferior a
12\%) durante a etapa de colheita da soja sobre a qualidade das sementes (Bunch, 1960; Costa et al., 1979; França Neto e Henning, 1984), contudo, neste trabalho, no horário de 18 horas, quando o grau de umidade mostrou-se inferior a este percentual para a cultivar BRS 184, devido às regulagens adequadas e específicas para a produção de sementes das colhedoras não foram verificadas diferenças entre as porcentagens de danos mecânicos.

Trabalhando com colhedoras de cilindro com côncavo, independentemente da regulagem, Costa et al. (1996) encontraram diferenças físicas e fisiológicas nas sementes de soja quando estas foram colhidas com umidade de $10,8 \%$.

A colhedora de cilindro com côncavo, deslocando-se a $5,0 \mathrm{~km} \cdot \mathrm{h}^{-1} \mathrm{e}$ a axial a $8,0 \mathrm{~km} \cdot \mathrm{h}^{-1}$, beneficiaram as sementes com resultados que não diferiram estatisticamente entre si (Tabela 1). Ressalta-se que a velocidade média da colhedora de 4,5km. $\mathrm{h}^{-1}$ tem sido usada normalmente para a colheita de sementes de soja (Costa et al., 1996).

As partes embrionárias da semente de soja são compostas de um tegumento pouco espesso, o qual lhe confere baixa proteção contra choques e abrasões que se verificam durante a colheita mecânica, comprometendo, na maioria das vezes, a qualidade fisiológica da semente (França Neto e Henning, 1984). Apesar da sensibilidade das sementes de soja às danificações mecânicas, não foi possível detectar diferenças fisiológicas nas análises realizadas, nos sistemas de colheita empregados no presente trabalho.

Embora não tenha sido evidenciada a diferença de comportamento entre as duas cultivares amostradas neste trabalho, Costa et al. (1996) consideram que esse é um aspecto importante a ser observado, pois existem diferenças genéticas entre as várias cultivares disponíveis no mercado.

Os valores correspondentes aos resultados de vigor

TABELA 1. Qualidade física e fisiológica de sementes de soja, cultivares BRS 184 e BRS 133, colhidas pelas colhedoras de cilindro com côncavo e axial.

\begin{tabular}{|c|c|c|c|c|c|c|}
\hline \multirow{3}{*}{ Características } & \multicolumn{3}{|c|}{ BRS 184} & \multicolumn{3}{|c|}{ BRS 133} \\
\hline & \multirow[b]{2}{*}{ C.V.\% } & \multicolumn{2}{|c|}{ Sistema } & \multirow[b]{2}{*}{ C.V.\% } & \multicolumn{2}{|c|}{ Sistema } \\
\hline & & Côncavo & Axial & & Côncavo & Axial \\
\hline Germinação & 2,43 & $93,22 \mathrm{~A}$ & $94,33 \mathrm{~A}$ & 2,57 & $89,08 \mathrm{~A}$ & $89,33 \mathrm{~A}$ \\
\hline Vigor (EA) & 5,14 & $86,89 \mathrm{~A}$ & $84,78 \mathrm{~A}$ & 7,11 & $86,42 \mathrm{~A}$ & $81,33 \mathrm{~A}$ \\
\hline Tetrazólio & 2,23 & $92,44 \mathrm{~A}$ & $93,22 \mathrm{~A}$ & 2,59 & $90,75 \mathrm{~A}$ & $89,92 \mathrm{~A}$ \\
\hline Dano (1) & 35,79 & $1,89 \mathrm{~A}$ & $3,22 \mathrm{~A}$ & 49,35 & $3,67 \mathrm{~A}$ & $2,25 \mathrm{~A}$ \\
\hline Dano (2) & 17,98 & $4,22 \mathrm{~A}$ & $3,78 \mathrm{~A}$ & 23,22 & $4,83 \mathrm{~A}$ & $3,83 \mathrm{~A}$ \\
\hline Umidade de Campo & 2,99 & $12,41 \mathrm{~A}$ & $12,25 \mathrm{~A}$ & 6,90 & $14,23 \mathrm{~A}$ & $13,59 \mathrm{~A}$ \\
\hline Umidade Laboratório & 1,84 & $10,62 \mathrm{~A}$ & $10,77 \mathrm{~A}$ & 3,05 & $10,84 \mathrm{~A}$ & $10,63 \mathrm{~A}$ \\
\hline
\end{tabular}

Dano 1 - Sementes sem dano

Dano 2 - Sementes com dano mecânico

${ }^{1}$ Médias seguidas de mesma letra na linha não diferem entre si pelo teste de Tukey $\mathrm{p}<0,05$

EA - envelhecimento acelerado. 
(envelhecimento acelerado), tetrazólio, dano mecânico (1) e (2) e grau de umidade de laboratório, para as cultivares BRS 184 e BRS 133, não apresentaram diferenças entre os dois tipos de colhedora empregados, quer seja pelo tipo cilindro côncavo ou pela axial, conforme as médias comparadas pelo teste de Tukey ao nível de 5\% de probabilidade (Tabela 2).
Observou-se, para as duas cultivares, que a umidade de campo variou entre os horários de 10 e 18 horas, sendo de dois pontos percentuais para a cultivar BRS 184 e de três pontos percentuais para a BRS 1333 (Tabela 2). Embora tenham ocorrido essas variações de umidade da semente no decorrer do dia, não foram observadas diferenças quanto às danificações mecânicas.

TABELA 2. Qualidade física e fisiológica de sementes de soja, colhidas nos horários de 10:00 horas, 14:00 horas e 18:00 horas.

\begin{tabular}{|c|c|c|c|c|c|c|c|c|}
\hline \multirow{3}{*}{ Características } & \multicolumn{4}{|c|}{ BRS 184} & \multicolumn{4}{|c|}{ BRS 133} \\
\hline & \multirow{2}{*}{ C.V.\% } & \multicolumn{3}{|c|}{ Horarios } & \multirow{2}{*}{ C.V.\% } & \multicolumn{3}{|c|}{ Horários } \\
\hline & & $10: 00$ & $14: 00$ & $18: 00$ & & $10: 00$ & $14: 00$ & $18: 00$ \\
\hline Germinação & 2,43 & $95,00 \mathrm{~A}_{1}$ & $93,33 \mathrm{~A}$ & $94,00 \mathrm{~A}$ & 2,57 & $89,00 \mathrm{~A}$ & $89,63 \mathrm{~A}$ & $89,00 \mathrm{~A}$ \\
\hline Vigor (EA) & 5,14 & $87,00 \mathrm{~A}$ & $85,83 \mathrm{~A}$ & $84,67 \mathrm{~A}$ & 7,11 & $82,88 \mathrm{~A}$ & $85,38 \mathrm{~A}$ & $83,38 \mathrm{~A}$ \\
\hline Tetrazólio & 2,23 & $93,67 \mathrm{~A}$ & $92,17 \mathrm{~A}$ & $92,67 \mathrm{~A}$ & 2,59 & $91,50 \mathrm{~A}$ & $90,50 \mathrm{~A}$ & $89,00 \mathrm{~A}$ \\
\hline Dano (1) & 35,79 & $3,17 \mathrm{~A}$ & $2,00 \mathrm{~A}$ & $2,50 \mathrm{~A}$ & 49,35 & $2,75 \mathrm{~A}$ & $2,38 \mathrm{~A}$ & $3,75 \mathrm{~A}$ \\
\hline Dano (2) & 17,98 & $3,00 \mathrm{~A}$ & $4,83 \mathrm{~A}$ & $4,17 \mathrm{~A}$ & 23,22 & $3,25 \mathrm{~A}$ & $4,13 \mathrm{~A}$ & $5,63 \mathrm{~A}$ \\
\hline Umidade de Campo & 2,99 & $13,33 \mathrm{~A}$ & $12,18 \mathrm{~B}$ & $11,48 \mathrm{C}$ & 6,90 & $15,50 \mathrm{~A}$ & $13,98 \mathrm{~B}$ & $12,26 \mathrm{C}$ \\
\hline Umidade Laboratório & 1,84 & $10,77 \mathrm{~A}$ & $10,65 \mathrm{~A}$ & $19,67 \mathrm{~A}$ & 3,05 & $10,79 \mathrm{~A}$ & $10,66 \mathrm{~A}$ & $10,76 \mathrm{~A}$ \\
\hline
\end{tabular}

Dano 1 - Sementes sem dano

Dano 2 - Sementes com dano mecânico

${ }^{1}$ Médias seguidas de mesma letra não diferem entre si pelo teste de Tukey $(\mathrm{p}<0,05)$.

EA - envelhecimento acelerado.

\section{CONCLUSÕES}

Há variações no grau de umidade da semente de soja durante o dia, podendo, ao final da tarde (18 horas), apresentar-se inferior ao início da colheita (10 horas), em até três pontos percentuais.

Tanto a colhedora de cilindro côncavo como a axial, desde que convenientemente utilizadas na colheita quanto às especificações de regulagem, não provocam diferenças na qualidade fisiológica da semente de soja.

\section{AGRADECIMENTOS}

Os autores agradecem a Lino Favoreto e Filhos Sementes Eldorado, por propiciar as condições necessárias para a execução deste trabalho.

\section{REFERÊNCIAS}

BANZATTO, D. A.; KRONKA, S.N. Experimentação Agrícola. Jaboticabal: Fundação de Estudo e Pesquisa em Agronomia, Medicina Veterinária e Zootecnia, 1992. 247p.

BRASIL, Ministério da Agricultura e da Reforma Agrária. Regras para análise de sementes. Brasília: SNDA/DNDV/CLAV, 1992. 365p.
BUNCH, H.D. Relationships between moisture content of seed and mechanical damage in seed conveying. Seed World, Chicago, v.86, n.5, p.14, 16-17, 1960.

CARVALHO, N.M.; NAKAGAWA, J. Sementes: ciência, tecnologia e produção. 4 ed. Jaboticabal: FUNEP, 2000. 588p.

COSTA, N.P., MESQUITA, C.M.; HENNING, A.A. Avaliação das perdas e qualidade de semente na colheita mecânica da soja. Revista Brasileira de Sementes, Brasília. v.1, n.3, p.59-70, 1979.

COSTA, N.P.; OLIVEIRA, M.C.N.; HENNING, A.A.; KRZYZANOWSKI, F.C.; MESQUITA, C.M.; TAVARES, L.C.V. Efeito da colheita mecânica sobre a qualidade de semente de soja. Revista Brasileira de Sementes, Brasília, v.18, n.2, p.232-237, 1996.

COSTA, N.P.; FRANÇA-NETO, J.B.; PEREIRA, J.E.; MESQUITA, C.M.; KRZYZANOWSKI, F.C.; HENNING, A. A. Efeito de sementes verdes na qualidade fisiológica de semente de soja. Revista Brasileira de Sementes, Brasília, v.23, n.2, p.102-107, 2001.

EMPRESA BRASILEIRA DE PESQUISA AGROPECUÁRIA. Tecnologias de produção de soja. Londrina, 2002. 195p.

FRANÇA NETO, J.B.; HENNING, A. A. Qualidade fisiológica da semente. Londrina: EMBRAPA CNPSo, 1984. p.5-24. (Circular Técnica, 9).

FRANÇA NETO, J.B.; KRZYZANOWSKI, F.C.; COSTA, N.P. Teste de tetrazólio em sementes de soja. Londrina: EMBRAPA CNPSo, 1998. 72p. (Documentos, 116).

MARCOS FILHO, J.; CÍCERO, S.M.; SILVA, W.R. Avaliação da qualidade fisiológica de sementes. Piracicaba: FEALQ, 1987. 230p.

MARCOS FILHO, J.; CÍCERO, S.M.; TOLEDO, F.F. Manual de 
análise de sementes. Piracicaba: ESALQ, 1985. 112p.

MARCOS, S.; MIELII, U. Axial-Flow, a mais nova campeã de produtividade, Disponível em <http://www.sppress.net/E-Foco>. Acesso em 26/05/2003.

PAIVA, L.E.; MEDEIROS, S.F.; FRAGA, A.C. Beneficiamento de sementes de milho colhidas mecanicamente em espigas: efeitos sobre danos mecânicos e qualidade fisiológica. Ciência Agrotécnica, Lavras, v.24, p.846-856, 2000.

PARANÁ. Secretaria de Estado da Agricultura e do Abastecimento/ Departamento de Fiscalização. Dados de Produção de Sementes: safra 2001/2002. Curitiba, 2003. 57p.

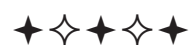

